\title{
The relation of omentin gene expression and glucose homeostasis of visceral and subcutaneous adipose tissues in non-diabetic adults
}

\section{Afsoon Daneshafrooz}

Research Institute for Endocrine Sciences

\section{Emad Yuzbashian}

Research Institute for Endocrine Sciences

Maryam Zarkesh ( $\nabla$ maryamzarkesh@yahoo.com )

Research Institute for Endocrine Sciences

\section{Golaleh Asghari}

Research Institute for Endocrine Sciences

\section{Parvin Mirmiran}

Research Institute for Endocrine Sciences

\section{Mehdi Hedayati}

Research Institute for Endocrine Sciences

\section{Raziyeh Abooshahab}

Research Institute for Endocrine Sciences

\section{S. Melika Fanaei}

Shahid Beheshti University of Medical Sciences

\section{Alireza Khalaj}

Shahed University

\section{Research Article}

Keywords: omentin expression, adipose tissues, glucose homeostasis, insulin resistance

Posted Date: May 3rd, 2021

DOl: https://doi.org/10.21203/rs.3.rs-326204/v1

License: (c) (i) This work is licensed under a Creative Commons Attribution 4.0 International License. Read Full License 


\section{Abstract \\ Background}

Adipose tissue (AT) is known as a passive reservoir for energy storage and an active endocrine organ responsible for the synthesis of bioactive molecules called adipokines. Omentin is known as an antiinflammatory adipokine that can modulate insulin sensitivity. In the present study, we aimed to investigate the relationship between omentin mRNA expression and glucose homeostasis of visceral adipose tissue (VAT) and subcutaneous adipose tissue (SAT) in non-diabetic adults.

\section{Methods}

VAT and SAT adipose tissues were collected from 137 adults, aged $\geq 18$ years, who were hospitalized for abdominal surgery. Preoperative venous blood samples were taken from the participants before surgery to measure fasting plasma glucose, insulin, and triglyceride. BMI, HOMA-IR, and HOMA-B were calculated. Insulin levels were measured with Mercodia kits using enzyme-linked immunosorbent assay (ELISA). In order to obtain omentin mRNA expression, real-time PCR was performed.

\section{Results}

Overall, 91 (51.7\%) subjects were healthy (without insulin resistance (IR)), and 46 (26.1\%) participants were with IR. Fold changes of VAT and SAT omentin expression in IR subjects were 2.32 and 1.30 , respectively $(P>0.05)$. After controlling for age and $B M I$, linear regression analysis indicated a significant positive association of SAT omentin expression with insulin concentration ( $\beta=0.048 ; 95 \% \mathrm{Cl}$ : 0.009 , $0.088, P=0.017)$ and HOMA-IR $(\beta=0.173 ; 95 \% \mathrm{Cl}: 0.023,0.323, P=0.014)$. Moreover, a negative association of SAT omentin expression with HOMA-B $(\beta=-0.001 ; 95 \% \mathrm{Cl}: 0.002,-0.001, \mathrm{P}<0.001)$ was observed.

\section{Conclusion}

This study's finding confirms a direct association between IR with omentin mRNA levels in SAT. Besides, the indicator of insulin sensitivity had an inverse association with omentin gene expression in SAT. This aspect of research suggests that omentin secretion from SAT has a strong link with insulin regulation.

\section{Introduction}

Adipose tissue (AT) is considered as an active endocrine organ due to its role in the body's homeostasis by secretion of bioactive mediators called adipokines with endocrine, paracrine hormonal functions [1]. It has also been well shown that these bioactive mediators derived from fat cells play a vital role in both insulin and energy homeostasis [2]. There are two types of AT in mammals: white adipose tissue (WAT) 
and brown adipose tissue (BAT). White adipose tissue is classified into two groups: visceral adipose tissue (VAT) and subcutaneous adipose tissue (SAT) [3]. Excessive fat alters AT metabolism and endocrine function, contributing to adipokine levels, thereby affecting insulin sensitivity and regulating energy homeostasis throughout the body [4]. The increased abdominal fat causes the secretion of inflammatory activating peptides, or adiponectin, from adipose tissue $[5,6]$. These adipokines have critical effects on glucose and lipid metabolism [7], insulin resistance (IR), diabetes, and so on [8].Nowadays, novel adipokines related to AT are important research topics. There are various types of adipokines, including adiponectin, resistin, leptin, visfatin, TNFa, and IL-6 [9-11]. Omentin gene was initially investigated by Yang et al. (2003) from the VAT cDNA library [12]. Omentin is a secretory protein with 313 amino acids mainly expressed in VAT adipose tissue. This protein, also known as the most potent anti-inflammatory adipokine, acts as a modulator of glucose metabolism by increasing insulinmediated glucose uptake and stimulating human body fat [12]. Indeed, omentin can increase insulin sensitivity and glucose metabolism in VAT through the paracrine and endocrine manner in which protein kinase (Akt/protein kinase $B$ ) is activated, thereby insulin signal transduction increased $[13,14]$. This signal is derived from fat cells that directly bind to adiponectin, acting as an insulin sensitizer [15]. Omentin can accelerate insulin-mediated glucose transport and does not affect basal glucose transport [12]. Removal of VAT instead of the SAT has been shown to improve insulin sensitivity [16]. Hence, the focus of studies on the omentin gene expression in AT as the primary source of omentin biosynthesis can be warranted. Thus far, numerous studies have shown down-regulation of omentin in obesity, type 2 diabetes mellitus (T2DM), and IR [17-21]. In a previous study, which is carried out on patients with impaired glucose regulation (IGR), newly diagnosed and untreated T2DM patients, and subjects with normal glucose tolerance (NGT), revealed that omentin levels were adversely correlated with BMI, HOMAIR, fasting insulin, TNF-a, and IL-6. Moreover, plasma glucose, HOMA-IR, and BMI were independent related factors that influenced serum omentin levels, which was decreased in IGR subjects [19]. Data from another study showed a high expression of omentin mRNA in VAT. On the other hand, the expression of omentin mRNA was decremented in individuals who were obese as well as in a combination of obese/T2D [22]. A few studies evaluate the expression of omentin within VAT and SAT in non-diabetic patients with IR. Hence, this study attempts to examine the expression of the omentin gene expression in VAT and SAT of non-diabetic adults and then compares its expression with glucose homeostasis.

\section{Materials And Methods}

\section{Study population}

In this cross-sectional study, we enrolled 137 adults, aged range 18-84 years, who were hospitalized for abdominal surgery. According to their IR status, participants were divided into two groups, subjects with IR $(n=46)$ and healthy $(n=91)$. Participants who have cancer, diabetes mellitus, or received fat-reducing, anti-obesity, and blood sugar drugs were excluded. Moreover, individuals with pregnancy and lactation or hospitalization less than two days before surgery were also excluded. 
People's general characteristics, including sex, age, medical history, and history of drug use (hypoglycemic drugs, lipid-lowering drugs, hypertension drugs, heart drugs, hormonal drugs, supplements), were asked and recorded.

Blood samples, anthropometric information, and demographic characteristics were obtained before surgery. About $100 \mathrm{mg}$ of VAT and SAT was also collected during surgery. The study's protocol was approved by the Research Institute for Endocrine Sciences (RIES) of Shahid Beheshti University of Medical Sciences (NO: IR.SBMU.ENDOCRINE.REC.1395.279). All participants consciously signed the written consent form approved by the committee.

\section{Measurement of anthropometric parameters and blood pressure}

As described previously, the weight, height, waist circumference, and blood pressure of participants were assessed $[23,24]$. Body mass index (BMI) was calculated as weight $(\mathrm{kg})$ divided by the square of height $\left(m^{2}\right)$.

\section{Assessment of Biochemical and glucose homeostasis}

Preoperative and fasting venous blood samples were taken from the participants and poured into potassium-EDTA tubes. Fasting plasma glucose (FPG) was measured by the enzymatic method of glucose oxidase. The enzyme colorimetric method with glycerol phosphate oxidase was also used to measure triglycerides (TGs). FPG and TGs were measured using commercial kits (Pars Azmoon Inc., Tehran, Iran). Insulin levels were measured with Mercodia kits (Uppsala, Sweden) using enzyme-linked immunosorbent assay (ELISA). The intra- and inter-test CVs were 1.7 and 2.3\%, respectively.

\section{RNA extraction and quantitative real-time polymerase chain reaction (qRT-PCR)}

Both adipose tissues were isolated by biopsy and collected in RNAlater solution. After transfer to the laboratory, the RNAlater solution was removed, and samples were placed in liquid nitrogen and then stored at $-80^{\circ} \mathrm{C}$. We extracted total RNA from both adipose tissues using the TRIzol reagent (Invitrogen U.S Cat. No. 15596-026) according to the manufacturer's protocol. The quantity and purity of RNA were evaluated by NanoDrop spectrophotometer (Thermo Fisher Scientific, Waltham, USA), and the absorption ratio $(260 / 280 \mathrm{~nm})$ of all samples were within an acceptable range. In order to eliminate genomic traces and increase purity, total RNA was treated with DNase I before the synthesis of complementary DNA (cDNA). The cDNA synthesis kit (BioFact, Korea) was used according to the manufacturer's protocol. GAPDH gene was also used as a reference gene to normalize omentin gene expression. The sequence of primers is shown in Table 1.

The Quantitative Reverse Transcriptase-PCR (qRT-PCR) amplification was performed in a $20 \mu \mathrm{l}$ reaction volume by the SYBR Green master mix (Biofact, South Korea) was done using the Rotor-Gene 6000 device (R Corbett Research, Sydney, Australia). The following thermal cycling included initial denaturation $\left(5 \mathrm{~min}\right.$ at $95^{\circ} \mathrm{C}$ ), followed by 45 cycles of $30 \mathrm{~s}$ at $95^{\circ} \mathrm{C}, 30 \mathrm{~s}$ at $60^{\circ} \mathrm{C}$, and $30 \mathrm{~s}$ at $72{ }^{\circ} \mathrm{C}$. For each gene, 
samples were run in duplicate for inter-assay control with the GAPDH reference gene and the nontemplate control (NTC). Relative expression of the omentin gene in each sample calculated by the $2^{-D C T}$ method based on its threshold cycle (Ct), the reference gene was used to normalize CT [25]. All qPCR laboratory steps were written according to the MIQE guidelines [26].

We used the following formulas to calculate HOMA-IR, HOMA-B, and QUICKI:

HOMA-IR= [fasting insulin $\mu \mathrm{U} / \mathrm{mL}=$ fasting glucose $\mathrm{mmol} / 1$ )]/22. HOMA-IR stands for the evaluation of the homeostatic model of IR. Participants with HOMA-IR $\geq 3.2$ were categorized in the IR group.

HOMA-B $=20 \times$ fasting insulin $(\mu \mathrm{lU} / \mathrm{ml}) /$ fasting glucose $(\mathrm{mmol} / \mathrm{ml})-3.5$. HOMA-B stands for insulin sensitivity.

QUICKI= 1/(log [fasting insulin $\mu \mathrm{U} / \mathrm{l}]+\log$ [fasting glucose $\mathrm{mg} / \mathrm{dl}])$. QUICKI stands for quantitative insulin sensitivity check index.

\section{Statistical analysis}

The normal distribution of variables was evaluated by histogram and Kolmogorov-Smirnov test. Data analysis was cried out by Statistical Package for Social Sciences software (SPSS) (Chicago IL. Ver. 15). $\mathrm{P}<0.05$ was considered statistically significant. Continuous variables were reported as mean \pm standard deviation (SD). As plasma TGs and insulin were skewed, we reported median and inter-quartile ranges. Ttest and $x^{2}$ tests were used to compare demographic data, anthropometrical, and plasma biochemical parameters between IR and healthy subjects. Linear regression was performed to determine the association of glucose homeostasis and omentin expression in VAT and SAT, and standardized $\beta$ (STZ $\beta$ ), after adjusting for BMI and sex.

\section{Results}

\section{Study participants}

In IR and healthy subjects, the mean (SD) age of participants were 36.2(10.8) and 43.1(14.1) years, respectively. As presented in Table 2, participants with IR were younger than healthy groups $(P=0.001)$. It is apparent from this table that BMI, insulin, TG, and FPG concentration in IR subjects were significantly higher when compared with healthy ones $(P<0.05)$.

\section{Omentin gene expression in VAT and SAT}

From the data in Figure 1, we can see that VAT omentin mRNA expression has increased in IR compared to healthy individuals. Further, a slight decreased in the expression of omentin in SAT was observed. However, these differences were marginally significant.

\section{Omentin gene expression status and glucose homeostasis}


The $\beta$ coefficient for the relationship between omentin gene expression and glucose homeostasis parameter are presented in Table 3. After controlling for age and BMI, linear regression analysis indicated a significant positive association of omentin gene expression in SAT with insulin concentration ( $\beta=0.048$; $95 \% \mathrm{Cl}: 0.009,0.088, \mathrm{P}=0.017)$. There was also a significant positive correlation between omentin mRNA levels and HOMA-IR $(\beta=0.173 ; 95 \% \mathrm{Cl}: 0.023,0.323, P=0.014)$. However, in $\mathrm{SAT}$, omentin gene expression was negatively associated with HOMA-B $(\beta=-0.001 ; 95 \% \mathrm{Cl}: 0.002,-0.001, P<0.001)$. Glucose homeostasis indices had no significant association with VAT omentin gene expression.

\section{Discussion}

Omentin is a novel anti-inflammatory adipokine with insulin-sensitive effects, which is related to obesity, $I R$, and T2D. Indeed, omentin is a secretory factor that can increase insulin sensitivity through Akt protein kinase $B$ in visceral and subcutaneous adiposity, thereby increasing glucose uptake in human adipocytes via the GLUT4 carrier and glucose metabolism [12]. In addition, increasing insulin sensitivity, which modulated by omentin, TNF-a one of the IR stimulators, can be suppressed by activating NF-kb cells [27, 28]. Further, omentin up-regulates the expression of the adiponectin, which can induce insulin sensitivity [28].

Seeking to find the expression of omentin in adipose tissues such as visceral and subcutaneous and its relation with glucose hemostasis may help find novel therapeutic strategies for patients with IR. The present study was designed to determine the expression of omentin mRNA in tissue samples of patients with IR compared with healthy subjects. The results of this study indicate a slight increase and decrease of mRNA expression in VAT and SAT (respectively) of patients with IR compared to healthy subjects.

Moreover, it is interesting to note that a direct association between insulin concentration and HOMA-IR with omentin mRNA levels in SAT was found. While, the indicator of insulin sensitivity, namely, HOMA-B, had an inverse association with omentin gene expression in SAT. Therefore, it is possible to hypothesize that omentin regulation could cause a disturbance in the IR.

Commonly, it had been argued that omentin, directly and indirectly, has a connection with IR. Some studies have revealed that omentin levels were reduced in patients with impaired glucose tolerance and obesity $[15,19]$. Indeed, the findings of our study can support the previous research.

In an investigation, Tan et al. (2008) found that plasma omentin-1 levels, omentin-1 mRNA, and protein expression in VAT of overweight women with PCOS were decreased compared to healthy subjects. Furthermore, they showed that insulin and glucose substantially dose-dependently led to decreased omentin-1 mRNA expression and protein levels. Therefore, it is suggested that insulin and glucose are directly or indirectly involved in omentin regulation [20].

Several studies supported the role of omentin in insulin sensitivity $[15,20]$. However, according to a study conducted by Hosseinnejad et al., no significant relation was observed between omentin, fasting insulin, and IR [29] that is also in contrast with our findings. In accordance with previous studies, omentin seems 
to be independent of SAT but more associated with VAT $[12,27]$. However, our results advocate some earlier studies regarding the differences in the expression of omentin in VAT and SAT, but the relation of omentin expression in SAT of IR subjects and glucose hemostasis was more noticeable in comparison with VAT of cases with IR. Therefore, it is likely that such connections exist between omentin expression in SAT and insulin regulation rather than its expression in VAT. These results differ from some published studies on obese children that have demonstrated a negative correlation between IR and omentin [30, 31].

There are two possible explanations for this difference; first, these studies were conducted on children and obesity, but our study was based on two groups: insulin resistant and insulin sensitive as a healthy group. Second, different antibody affinities in test kits can justify inequalities.

\section{Conclusion}

This cross-sectional study extends our knowledge of omentin expression in adipose tissue and its relation with glucose hemostasis, which confirms previous findings and contributes additional evidence that suggests glucose homeostasis may have a strong association with omentin mRNA levels in SAT. Given the beneficial properties of this adipokine and its protective role in preventing IR and inflammation, a future comprehensive study can determine whether these associations are causal and also the mechanisms underlying omentin expression in SAT and IR.

\section{Declarations}

Funding

Not applicable.

Competing interest

The authors declare they have no conflict of interest.

Availability of data and materials

Not applicable.

Code availability

Not applicable.

Authors' contributions

$\mathrm{MH}, \mathrm{MZ}, \mathrm{PM}, \mathrm{GA}$ participated in the study design. AK participated in the study coordination and data collection. EY participated in the data analysis; AD participated in the manuscript writing. 
EY and RA performed the statistical analysis, drafted the manuscript, and organized findings per PRISMA guidelines for SMF. All authors contributed to the data analysis, drafting or revising the article, and approved the final version.

Ethics approval

Not applicable.

Consent to participate

Not applicable.

Consent for publication

Not applicable.

\section{Abbreviations}

AT: adipose tissue; VAT: visceral adipose tissue; SAT: subcutaneous adipose tissue; WAT: white adipose tissue; BAT: brown adipose tissue; BMI: body mass index; PCR: polymerase chain reaction; CT: threshold cycle; NF-KB: nuclear factor-Kb; IR: insulin resistance; T2DM: type 2 diabetes mellitus; IGR: impaired glucose regulation; NGT: normal glucose tolerance; FPG: Fasting plasma glucose; TGs: triglycerides; QUICKI: quantitative insulin sensitivity check index; insulin sensitivity; HOMA-IR : the evaluation of the homeostatic model of IR; HOMA-B: insulin sensitivity; STZ $\beta$ : standardized $\beta$; ELISA: enzyme-linked immunosorbent assay.

\section{References}

1. Wozniak SE, Gee LL, Wachtel MS and Frezza EE (2009) Adipose tissue: the new endocrine organ? A review article. Digestive diseases and sciences 54:1847-1856.

2. Lago F, Gómez R, Gómez-Reino JJ, Dieguez C and Gualillo O (2009) Adipokines as novel modulators of lipid metabolism. Trends in biochemical sciences 34:500-510.

3. Seale P, Conroe HM, Estall J, Kajimura S, Frontini A, Ishibashi J, Cohen P, Cinti S and Spiegelman BM (2011) Prdm16 determines the thermogenic program of subcutaneous white adipose tissue in mice. The Journal of clinical investigation 121:96-105.

4. McGown C, Birerdinc A and Younossi ZM (2014) Adipose tissue as an endocrine organ. Clinics in liver disease 18:41-58.

5. Tan BK, Adya R and Randeva HS (2010) Omentin: a novel link between inflammation, diabesity, and cardiovascular disease. Trends in cardiovascular medicine 20:143-148.

6. Rabe K, Lehrke M, Parhofer KG and Broedl UC (2008) Adipokines and insulin resistance. Molecular medicine 14:741-751. 
7. Després J-P, Lemieux I, Bergeron J, Pibarot P, Mathieu P, Larose E, Rodés-Cabau J, Bertrand OF and Poirier P (2008) Abdominal obesity and the metabolic syndrome: contribution to global cardiometabolic risk. Arteriosclerosis, thrombosis, and vascular biology 28:1039-1049.

8. Zhong X, Zhang H-y, Tan H, Zhou Y, Liu F-I, Chen F-q and Shang D-y (2011) Association of serum omentin-1 levels with coronary artery disease. Acta Pharmacologica Sinica 32:873-878.

9. Shah A, Mehta N and Reilly MP (2008) Adipose inflammation, insulin resistance, and cardiovascular disease. Journal of Parenteral and Enteral Nutrition 32:638-644.

10. Kershaw EE and Flier JS (2004) Adipose tissue as an endocrine organ. The Journal of Clinical Endocrinology \& Metabolism 89:2548-2556.

11. Fukuhara A, Matsuda M, Nishizawa M, Segawa K, Tanaka M, Kishimoto K, Matsuki Y, Murakami M, Ichisaka T and Murakami H (2005) Visfatin: a protein secreted by visceral fat that mimics the effects of insulin. Science 307:426-430.

12. Yang R-Z, Lee M-J, Hu H, Pray J, Wu H-B, Hansen BC, Shuldiner AR, Fried SK, McLenithan JC and Gong D-W (2006) Identification of omentin as a novel depot-specific adipokine in human adipose tissue: possible role in modulating insulin action. American Journal of Physiology-Endocrinology and Metabolism 290:E1253-E1261.

13. Rostami H, Samadi M, Yuzbashian E, Zarkesh M, Asghari G, Hedayati M, Daneshafrooz A, Mirmiran P and Khalaj A (2017) Habitual dietary intake of fatty acids are associated with leptin gene expression in subcutaneous and visceral adipose tissue of patients without diabetes. Prostaglandins, Leukotrienes and Essential Fatty Acids 126:49-54.

14. Yuzbashian E, Zarkesh M, Asghari G, Hedayati M, Safarian M, Mirmiran P and Khalaj A (2018) Is apelin gene expression and concentration affected by dietary intakes? A systematic review. Critical reviews in food science and nutrition 58:680-688.

15. de Souza Batista CM, Yang R-Z, Lee M-J, Glynn NM, Yu D-Z, Pray J, Ndubuizu K, Patil S, Schwartz A and Kligman M (2007) Omentin plasma levels and gene expression are decreased in obesity. Diabetes 56:1655-1661.

16. Thörne A, Lönnqvist F, Apelman J, Hellers G and Arner P (2002) A pilot study of long-term effects of a novel obesity treatment: omentectomy in connection with adjustable gastric banding. International journal of obesity 26:193-199.

17. Shibata R, Ouchi N, Takahashi R, Terakura Y, Ohashi K, Ikeda N, Higuchi A, Terasaki H, Kihara S and Murohara T (2012) Omentin as a novel biomarker of metabolic risk factors. Diabetology \& metabolic syndrome 4:1-4.

18. Auguet T, Quintero Y, Riesco D, Morancho B, Terra X, Crescenti A, Broch M, Aguilar C, Olona M and Porras JA (2011) New adipokines vaspin and omentin. Circulating levels and gene expression in adipose tissue from morbidly obese women. BMC medical genetics 12:1-8.

19. Pan H-Y, Guo L and Li Q (2010) Changes of serum omentin-1 levels in normal subjects and in patients with impaired glucose regulation and with newly diagnosed and untreated type 2 diabetes. Diabetes research and clinical practice 88:29-33. 
20. Tan BK, Adya R, Farhatullah S, Lewandowski KC, O'Hare P, Lehnert H and Randeva HS (2008) Omentin-1, a novel adipokine, is decreased in overweight insulin-resistant women with polycystic ovary syndrome: ex vivo and in vivo regulation of omentin-1 by insulin and glucose. Diabetes 57:801808.

21. El-Mesallamy H, El-Derany M and Hamdy N (2011) Serum omentin-1 and chemerin levels are interrelated in patients with Type 2 diabetes mellitus with or without ischaemic heart disease. Diabetic Medicine 28:1194-1200.

22. Cai R, Wei L, Di J, Yu H, Bao Y and Jia W (2009) Expression of omentin in adipose tissues in obese and type 2 diabetic patients. Zhonghua yi xue za zhi 89:381-384.

23. Nosrati-Oskouie M, Yuzbashian E, Zarkesh M, sadat Aghili-Moghadam N, Hedayati M, Safarian M, Asghari G, Khalaj A and Mirmiran P (2020) Association of plasma fatty acids pattern with omentin gene expression in human adipose tissues: A cross-sectional study. Nutrition, Metabolism and Cardiovascular Diseases.

24. Yuzbashian E, Asghari G, Hedayati M, Zarkesh M, Mirmiran P and Khalaj A (2019) Determinants of vitamin $D$ receptor gene expression in visceral and subcutaneous adipose tissue in non-obese, obese, and morbidly obese subjects. The Journal of steroid biochemistry and molecular biology 187:82-87.

25. Livak KJ and Schmittgen TD (2001) Analysis of relative gene expression data using real-time quantitative PCR and the 2- $\triangle \Delta C T$ method. methods 25:402-408.

26. Bustin SA, Benes V, Garson JA, Hellemans J, Huggett J, Kubista M, Mueller R, Nolan T, Pfaffl MW and Shipley GL (2009) The MIQE Guidelines: M inimum I nformation for Publication of Q uantitative RealTime PCR E xperiments. Oxford University Press,

27. Yamawaki H, Tsubaki N, Mukohda M, Okada M and Hara Y (2010) Omentin, a novel adipokine, induces vasodilation in rat isolated blood vessels. Biochemical and biophysical research communications 393:668-672.

28. Reinehr T and Roth CL (2018) Inflammation markers in type 2 diabetes and the metabolic syndrome in the pediatric population. Current diabetes reports 18:131.

29. Hossein-Nezhad A, Mirzaei K, Alatab S, Ahmadivand Z, Najmafshar A, Peppa M, Koliaki C, Dimitriadis G, Sivakumar T and Sivakumar S (2012) Circulating omentin-1 in obesity and metabolic syndrome status compared to control subjects. Endocrinol Metab Syndr 1:008.

30. Buyukinan M, Atar M, Can U, Pirgon O, Guzelant A and Deniz I (2018) The association between serum vaspin and omentin-1 levels in obese children with metabolic syndrome. Metabolic Syndrome and Related Disorders 16:76-81.

31. Zengi S, Zengi O, Kirankaya A, Kucuk SH, Kutanis EE and Yigit O (2019) Serum omentin-1 levels in obese children. Journal of Pediatric Endocrinology and Metabolism 32:247-251.

\section{Tables}

Jle1. Sequences and information of Omentin and GAPDH primers for PCR reaction. 


\begin{tabular}{ccccccc}
\hline Genes & & Primers' sequences 5'-3' $^{\prime}$ & Length & Tm & GC\% & product length (pb) \\
\hline Omentin & F: & AGGAGCTCTCTGTACCCAAG & 20 & 58.14 & 55 & 119 \\
& R: & TAGCCTCATCTGTACTCCATC & 21 & 56.05 & 47.62 & \\
\multirow{2}{*}{ GAPDH } & F: & CTGCTCCTCCTGTTCGACAGT & 21 & 61.76 & 57.14 & 100 \\
& R: & CCGTTGACTCCGACCTTCAC & 20 & 60.67 & 60 & \\
\hline
\end{tabular}

able2. Demographic, anthropometric and serum biochemical parameters of the studied opulation.

\begin{tabular}{lccc}
\hline \multicolumn{1}{c}{ Variables } & $\begin{array}{c}\text { Healthy } \\
(\mathrm{n}=91)\end{array}$ & $\begin{array}{c}\text { Insulin resistance } \\
(\mathrm{n}=46)\end{array}$ & $P$ value \\
\hline Age (years) & $43.9 \pm 14.1$ & $35.7 \pm 10.4$ & $<0.001$ \\
Female (\%) & 73.8 & 77.8 & 0.637 \\
\hline Body mass index $\left(\mathrm{Kg} / \mathrm{m}^{2}\right)$ & $33.6 \pm 10.5$ & $40.1 \pm 8.4$ & $<0.001$ \\
\hline Insulin $(\mathrm{\mu U} / \mathrm{mL})$ & $5.7(3.0-9.3)$ & $23.5(20.2-28.0)$ & $<0.001$ \\
\hline Triglycerides $(\mathrm{mg} / \mathrm{dl})$ & $84.5(66.0-144.5)$ & $119.5(80.5-155.8)$ & 0.020 \\
\hline Fasting plasma glucose $(\mathrm{mg} / \mathrm{dL})$ & $83.7 \pm 16.2$ & $99.7 \pm 21.4$ & $<0.001$ \\
\hline HOMA-IR & $1.33 \pm 0.8$ & $6.2 \pm 3.2$ & $<0.001$ \\
\hline HOMA-B & $85.5(39.4-141.9)$ & $251.5(198.1-382.1)$ & 0.764 \\
\hline QUIKI & $0.16 \pm 0.2$ & $0.13 \pm 0.0$ & $<0.001$ \\
\hline
\end{tabular}

tble3. Association between Omentin gene expression in VAT and SAT and glucose meostasis

\begin{tabular}{|c|c|c|c|c|c|c|}
\hline & \multicolumn{3}{|c|}{ Visceral } & \multicolumn{3}{|c|}{ Subcutaneous } \\
\hline & $\begin{array}{c}\text { confidence } \\
\text { interval } \\
\beta(95 \% \mathrm{CI})\end{array}$ & STZ $\beta$ & $\begin{array}{c}P \\
\text { value }\end{array}$ & $\begin{array}{c}\text { confidence } \\
\text { interval } \\
\beta(95 \% \mathrm{CI})\end{array}$ & STZ $\beta$ & $P$ value \\
\hline $\begin{array}{l}\text { Fasting plasma } \\
\text { glucose }\end{array}$ & $\begin{array}{c}0.023(-0.003 \\
0.049)\end{array}$ & 0.139 & 0.077 & $\begin{array}{c}-0.002(-0.024, \\
0.021)\end{array}$ & -0.011 & 0.891 \\
\hline Insulin & $\begin{array}{c}-0.033(-0.080 \\
0.014)\end{array}$ & -0.115 & 0.171 & $\begin{array}{c}0.048(0.009 \\
0.088)\end{array}$ & 0.2 & 0.017 \\
\hline HOMA-IR & $\begin{array}{c}-0.083(-0.259 \\
0.094)\end{array}$ & -0.076 & 0.356 & $\begin{array}{c}0.173(0.023 \\
0.323)\end{array}$ & 0.287 & 0.014 \\
\hline HOMA-B & $\begin{array}{c}-0.000 \\
(-0.001,0.001)\end{array}$ & -0.003 & 0.974 & $\begin{array}{c}-0.001(0.002, \\
-0.001)\end{array}$ & -0.436 & $<0.001$ \\
\hline QUICKI & $\begin{array}{c}15.82(-6.57 \\
38.22)\end{array}$ & 0.129 & 0.165 & $\begin{array}{c}-7.47(-26.79 \\
11.85)\end{array}$ & -0.071 & 0.446 \\
\hline
\end{tabular}



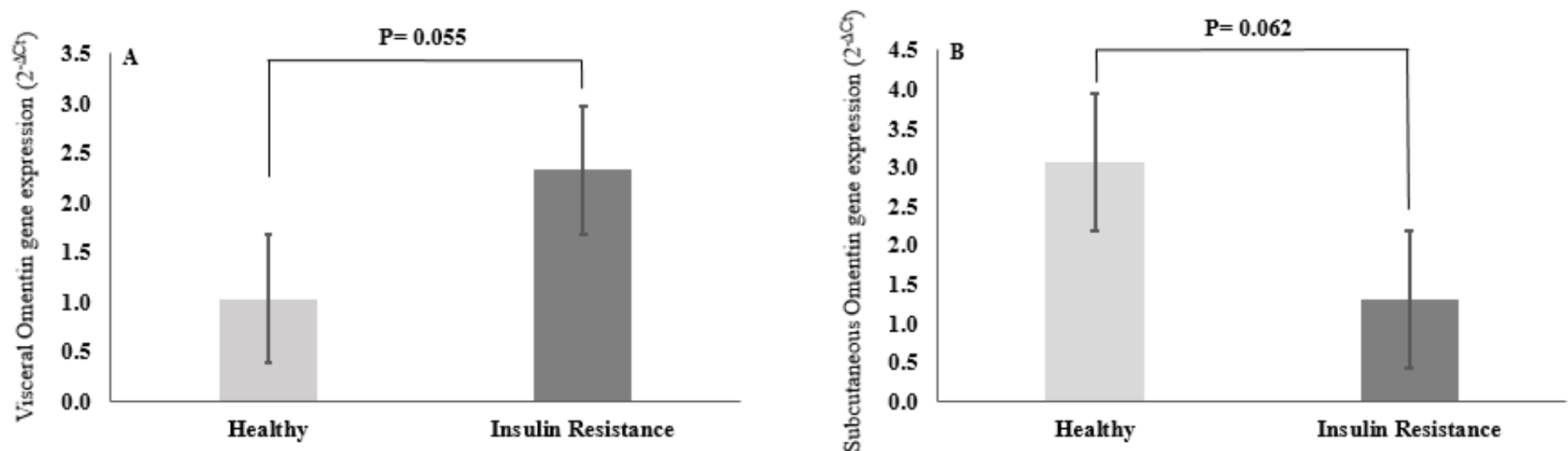

\section{Figure 1}

Mean and Standard Error for A) Visceral adipose tissue and B) Subcutaneous adipose tissue Omentin gene expression according to the presence of insulin resistance. Results are expressed as mean $\pm \mathrm{SEM}$. 